\title{
Impact of nuclear inertia momenta on fission observables
}

\author{
P. Tamagno* and O. Litaize \\ CEA, DEN, DER, SPRC, Cadarache, 13108 Saint-Paul-lez-Durance, France
}

\begin{abstract}
Fission is probably the nuclear process the less accurately described with current models because it involves dynamics of nuclear matter with strongly coupled manybody interactions. It is thus difficult to find models that are strongly rooted in good physics, accurate enough to reproduce target observables and that can describe many of the nuclear fission observables in a consistent way. One of the most comprehensive current modeling of the fission process relies on the fission sampling and Monte-Carlo de-excitation of the fission fragments. This model is implemented for instance in the FIFRELIN code. In this model fission fragments and their state are first sampled from pre-neutron fission yields, angular momentum distribution and excitation energy repartition law then the decay of both initial fragments is simulated. This modeling provides many observables: prompt neutron and gamma fission spectra, multiplicities and also fine decompositions: number of neutrons emitted as a function of the fragment mass, spectra per fragments, etc. This model relies on nuclear structure databases and on several basic nuclear models describing for instance gamma strength functions or level densities. Additionally some free parameters are still to be determined, namely two parameters describing the excitation energy repartition law, the spin cutoff of the heavy and light fragments and a rescaling parameter for the rotational inertia momentum of the fragments with respect of the rigid-body model. In the present work we investigate the impact of this latter parameter. For this we mainly substitute the corrected rigid-body value by a quantity obtained from a microscopic description of the fission fragment. The independent-particle model recently implemented in the CONRAD code is used to provide nucleonic wave functions that are required to compute inertia momenta with an Inglis-Belyaev cranking model. The impact of this substitution is analyzed on different fission observables provided by the FIFRELIN code.
\end{abstract}

\section{Introduction}

Nuclear moments of inertia have been very helpful since the early days of nuclear physics as a convenient tool to interpret several observables. Even if moments of inertia are more a nuclear property than a nuclear model itself it is widely used today as model ingredient. For instance as a parameter in a phenomenological parameterization carrying a more subtle dependency than a simple function of $A$. Its connection to macroscopic nuclear properties such as the nucleus size and shape make the moment of inertia a representative candidate for such parameters whenever one is looking for a parameter sensitive to global nuclear structure properties.

In the present work we highlight the presence and role of moments of inertia in well established and widely used models. We restrict our discussion to fission observables although a similar approach can be undertaken for other type of observables e.g. cross sections. In a first section we recall the usual classical and semi-classical models for nuclear moment of inertia. Then in a second section we present the cranking model based on the Finite-Range Liquid-Drop-Model [1] that we have implemented to provide moments of inertia carrying a more refined mark of the nuclear structure. In a third section we

*e-mail: pierre.tamagno@cea.fr 
show where these phenomenological "ingredients" are used in practical modeling of fission observables. The modeling scheme of the FIFRELIN code [2] will be used as an example. Finally the impact of the choice of model for nuclear moments of inertia will be illustrated on selected fission observables provided by the FIFRELIN code.

\section{Classical and semi-classical moments of inertia}

The dimensions of nucleons and nuclei imply that quantum mechanics must be used to describe comprehensively the dynamics of such objects. Yet classical moments of inertia remain in use today to provide at least order of magnitudes, or parameters for various systematics for instance in the RIPL library [3]. In classical mechanics, the moment of inertia $I_{z}$ about the $z$-axis for an object with a mass density $\rho$ is given by

$$
I_{z}=\int \mathrm{d}^{3} \mathbf{r}\left(r^{2}-z^{2}\right) \rho(\mathbf{r})
$$

It can be expected from Eq. 1 that $I_{z}$ will depend on the global nuclear structure properties through the mass density $\rho$. A shape-edge surface with an homogeneous mass distribution is usually assumed and justified by the short-range of nuclear interactions. With this additional hypothesis this model is known as the rigid body model. The rigid body moment of inertia only depends on the nucleus mass (approximatively, the number of nucleons) and the macroscopic shape of the nucleus. Expressions for Yukawa-folded densities have been derived [4] and showed to slightly increase the value of the moment of inertia compared to the rigid-body value. As long as only ground-state shapes are considered, one usually assumes that the macroscopic shape of the nucleus has a rotational invariance about the $z$-axis. In the spherical coordinate system, one can thus describe the nuclear radius using an expansion on Legendre polynomials, as:

$$
R(\theta, \varphi)=\frac{R_{0}}{\lambda\left(\beta_{2}, \beta_{3}, \ldots\right)}\left(1+\sum_{\ell=1} \beta_{\ell} \sqrt{\frac{2 \ell+1}{4 \pi}} P_{\ell}(\cos \theta)\right) .
$$

In Eq. $2, R_{0}$ is the radius of the nucleus for a spherical shape, $\lambda$ is a parameter that must be adjusted to keep a constant nuclear volume for different shapes (the nuclear matter is additionally assumed to be incompressible). Finally $\beta_{1}$ is also adjusted to keep the description in the center of mass of the nucleus. With the shape parameterization $R(\theta, \varphi)$, one can compute Eq. 1 (applied for an axis perpendicular to the symmetry axis) either numerically or using a power expansion in $\beta_{2}$. In such a case one obtains [5]:

$$
I_{\perp}^{\text {rigid-body }}=\frac{2}{5} M R_{0}^{2}\left(1+0.31 \beta_{2}+0.44 \beta_{2}^{2}+\ldots\right) .
$$

Here $M$ is the mass of the nucleus, it can also be replaced by $A$ times the average mass of a nucleon. In our calculations we observed that the last term in Eq. 3 amounts to a few percents of the total value while using usual tabulated ground state shape parameters [1]. This correction is slightly larger than the remaining difference with the numerical calculation carrying all orders in all $\beta$. An alternative approach is to consider the nuclear matter as an irrotational fluid. In this case the moment of inertia reads [5]

$$
I_{\perp}^{\text {irrotational }}=\frac{9}{8 \pi} A M R_{0}^{2} \beta_{2}^{2} .
$$

Such an approach is not investigated further here as it yields very small values that are inconsistent with nuclear spectroscopy results.

\section{Cranking model for nuclear moment of inertia}

A refined method consists in calculating the moment of inertia from a microscopic nuclear structure model. We have implemented [6] an Inglis-Belyaev cranking model [7,8] based on the Finite-Range 
Liquid-Drop-Model (FRLDM) [1]. In this model, the nuclear moment of inertia is not deduced from a macroscopic shape of a sharp-edge uniform volume but from single-particle wave functions of the nucleons. The FRLDM is a macroscopic-microscopic model, which means that the macroscopic shape is still used in order to parametrize the potential well (mean field) felt by the nucleons. Even if some improvements have been brought to the FRLDM since Ref. [1], the microscopic part of the model used to calculate the cranking moment of inertia remains identical. The cranking moment of inertia $I^{\text {cranking }}$ is the sum of the neutron and proton components $I^{(\mathrm{n})}$ and $I^{(\mathrm{p})}$. For instance for neutrons, the moment of inertia is given by

$$
I_{\perp}^{(\mathrm{n})}=2 \hbar^{2} \sum_{\nu \mu} \frac{\left|\left\langle v\left|j_{x}\right| \mu\right\rangle\right|^{2}}{E_{v}+E_{\mu}}\left(u_{\nu} v_{\mu}-u_{\mu} v_{v}\right)^{2},
$$

where $|v\rangle$ and $|\mu\rangle$ are the single-particle wave functions obtained with the Yukawa-folded independent particle model of the FRLDM. Energies $E_{v}$ and occupation probabilities $v_{v}^{2}$ are obtained from the independent particle states by applying either a BCS [9] or a Lipkin-Nogami $[10,11]$ pairing correlation correction. These developments where originally designed for the CONRAD evaluation code [12] but are now shared with FIFRELIN.

Before we investigate the impact of different models on nuclear moments of inertia, we make a comparison with experimental moments of inertia that can be extracted from spectroscopy measurements. To obtain experimental moments of inertia, the ENSDF database [13] has been automatically explored to find low lying $0^{+}, 2^{+}, 4^{+}$patterns in the level schemes. If the ratio of the two transition energies is close to $2(2+1) / 4(4+1)$ then a moment of inertia is extracted from the lower transition energy. In Fig. 1 the different models presented in this paper are compared with experimental data. The well-known overestimation by the rigid body model by about a factor of 2 and the dramatic underestimation by the irrotational model are clearly visible. The rigid body model has two kinds of structures, the mass dependency and the shape dependency, this latter being an indirect consequence of the nuclear quantum structure. The cranking model follows much more closely the trends of experimental data. This is a clear evidence that nuclear structure impacts the moment of inertia in a much deeper manner than just modifying the macroscopic nucleus shape.

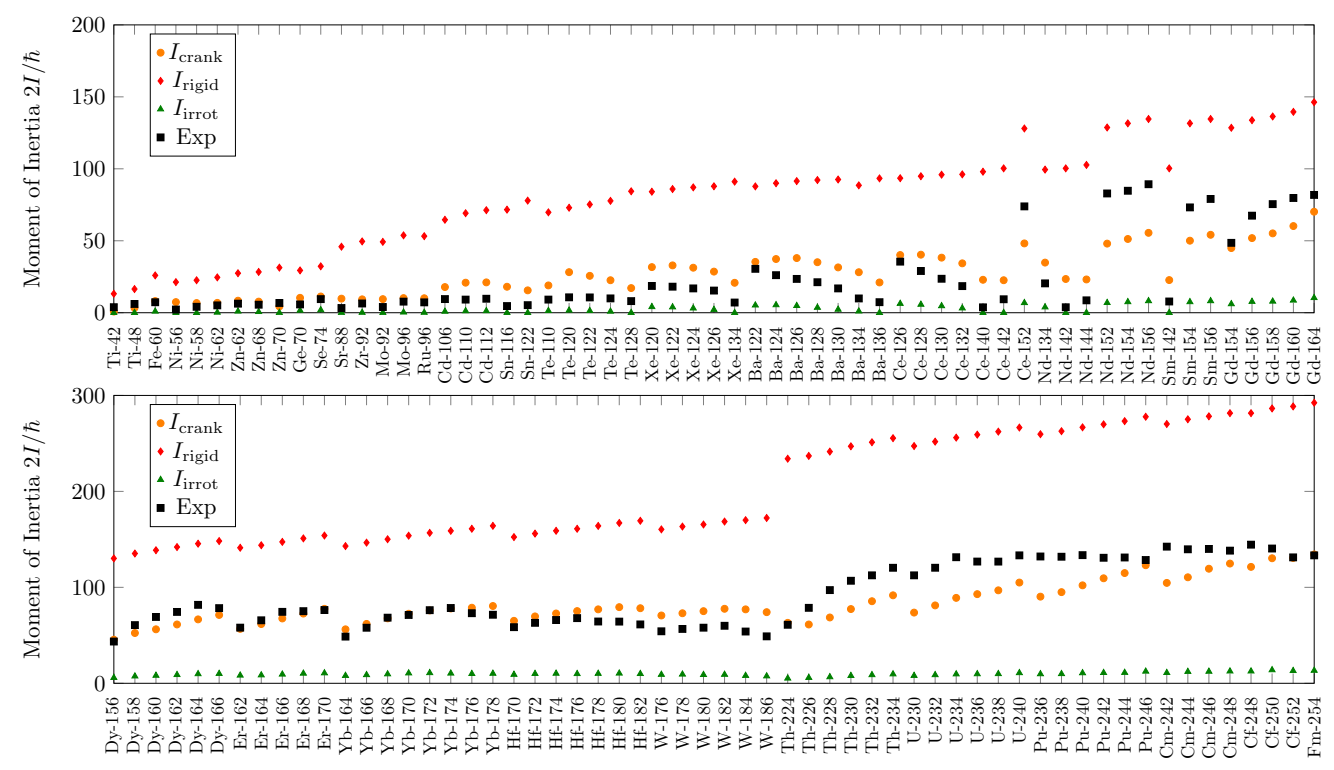

Fig. 1. Comparison of the different models for moment of inertia with experimental data. 


\section{Moments of inertia in nuclear models}

As mentioned in introduction, the moments of inertia are used as nuclear-structured parameters in systematics (phenomenological parameterizations). These systematics are obtained from broad analyses of experimental data in order to exhibit ad hoc trends or gross dependencies. The parameterization obtained can be used in turn to estimate not measured data and provides thus prior values of nuclear observables. Many nuclear models such as TALYS [14], CONRAD [12] or FIFRELIN [2] partly rely on phenomenological expressions for intermediate nuclear observables e.g. the nuclear level density. These intermediate observables can be provided by the phenomenological expressions. In the present work we will focus on two kinds of nuclear observable in which moments of inertia are involved: level densities and rotational energy. This latter is the easiest concept as the moment of inertia is involved in the classical or semi-classical description of the rotational energies. For a classical total angular momentum $\mathbf{J}$ the rotational energy of a rigid body is given by

$$
E_{\mathrm{rot}}=\frac{J_{z}^{2}}{2 I_{z}}+\frac{J_{y}^{2}}{2 I_{y}}+\frac{J_{x}^{2}}{2 I_{x}} .
$$

In case of axially symmetric nuclei, the rotation invariance can be interpreted as an infinite moment of inertia with respect of the $z$-axis, additionally one can write $I=I_{x}=I_{y}$, thus

$$
E_{\mathrm{rot}}=\frac{J_{x}^{2}+J_{y}^{2}}{2 I}=\frac{J^{2}-J_{z}^{2}}{2 I} .
$$

In case of a $K=0$ rotational band and for a quantified total angular momentum with $\mathbf{J}^{2}=J(J+1)$, it becomes

$$
E_{\mathrm{rot}}=\frac{J(J+1)}{2 I} .
$$

This expression occurs often in nuclear spectroscopy where low-lying levels of even-even nuclei follow the $0^{+}, 2^{+}, 4^{+}, \ldots$ rule. Equation 8 is also useful to determine the part of the excitation energy of a nucleus that is taken by collective degrees of freedom. This is used for instance in FIFRELIN. In FIFRELIN the fission observables are obtained in two-steps Monte Carlo simulations. Fist a fission event is sampled, determining the initial excitation states of the fission fragments. Then the decay of each fragment is simulated according to the Bečvár algorithm [15]. In the first step, the fragments mass numbers $\left(A_{\mathrm{L}}, A_{\mathrm{H}}\right)$ are first sampled from experimental fission yields, then their kinetic energies (KE) are also sampled from experimental data (knowing the mass number of each fragment). The total excitation energy $T X E$ can be calculated from the total released energy $Q$ by $T X E=Q-K E\left(A_{\mathrm{H}}\right)-$ $K E\left(A_{\mathrm{L}}\right)$. The total intrinsic excitation energy is given by $E^{*}=T X E-E_{\mathrm{L}}^{\text {rot }}-E_{\mathrm{H}}^{\text {rot }}+B_{\mathrm{n}}+E_{\mathrm{n}}^{\text {kin }}$, where $E_{\mathrm{L}}^{\text {rot }}$ and $E_{\mathrm{H}}^{\text {rot }}$ are respectively the rotational energies of the light and heavy fragments obtained with Eq. 8 . The total intrinsic excitation is then dispatched between the two fragments according to a temperature law [2].

Nuclear moments of inertia are also used in phenomenological expressions for spin cutoffs. Spin cutoffs $\sigma$ are introduced in the Fermi gas theory in order to describe the statistical distribution of total angular momentum of the gas. This model is used to describe the distribution of spins in a nucleus. It is for instance this type of law that is used in FIFRELIN to sample initial fragment spins or to attribute a spin of a level sampled in the continuum. In such an approach the probability that a given level is of total angular momentum $J$ is given by $[16,17]$

$$
P(J)=\frac{J+1 / 2}{\sigma^{2}} \exp \left[-\frac{(J+1 / 2)^{2}}{2 \sigma^{2}}\right]
$$

Expression 9 is close to a Rayleigh distribution; the energy dependency of the distribution is carried by the spin cutoff, which reads $\sigma^{2}=I \sqrt{U / a}$, where $U=E^{*}-\Delta$ is the available energy ( $\Delta$ is the pairing energy) and $a$ is the level density parameter [3]. It should be noted that expression 9 is not normalized. In practice a normalization coefficient close to unity must be applied to preserve the statistics. 


\section{Impact on fission observables}

We now investigate the impact of using the cranking-FRLDM model instead of the usual rigid body prescription. In FIFRELIN, five parameters must be tuned in order to reproduce target observables e.g. heavy and light fragments neutron multiplicities. These parameters are the spin cutoffs for the heavy and light fragments ${ }^{1}$, the fraction of the moment of inertia $k$ used in the rotational energy $\left(E_{\text {rot }}=\frac{J(J+1)}{2 k I}\right)$ and two parameters for the temperature law. The adjustment of these five parameters is cumbersome and time-consuming. We thus used a set of parameters fitted previously for the rigid body model. As the value for the rigid body model is about twice larger than the cranking model, we adjusted $k$ so that the order of magnitude is preserved. We attempt thus to see the impact of the structure of the cranking moment of inertia ( $c f$. Fig. 1) instead of the impact of the change in magnitude. We also did not change the prescription for the spin cutoffs for the sampling of level spin. Indeed we realized that the RIPL prescription [3] $\sigma^{2}=I^{\text {rigid }} a / \tilde{a} \sqrt{U / a}$, used in FIFRELIN was already altered in order to introduce shell correction in the spin cutoff.

Replacing $I^{\text {rigid }}$ by $I^{\text {cranking }}$ would count twice these effects. We thus tested the two sets of parameters for the ${ }^{252} \mathrm{Cf}$ spontaneous fission. In Fig. 2 one can see the impact of changing the moment of inertia for two fission observables provided by FIFRELIN. On the sawtooth, an surprising hump near $A=140$ appears. It is believed to be due to the transition in the counterpart fragment $(A=112)$ near the semi-magical number $N=70$ (see Fig 3). It is also interesting to notice that in the small mass region $(A<100)$ the cranking model is closer to the most recent measurements, whereas the rigid body values are closer to older experimental data. The impact on the average neutron energy can be seen in Fig. 2 as well. It can be observed that the gross structure in the region $120<A<140$ is well reproduced with the cranking model. Additionally the hump near $A=90$ that can be seen in the experimental data is reproduced (although with a too strong magnitude) with the cranking model only.

\section{Conclusion}

In this work, strong arguments are given for the use of moments of inertia based on a microscopic approach (FRLDM + cranking) instead of the usual rigid body model. The cranking model is in better agreement with data obtained from nuclear spectroscopy but also tends to have an impact on fission observables that is consistent with experimental data. In the future a more comprehensive study should be performed in order to obtain a new set of parameters that could reproduce the FIFRELIN target observables without relying on previously established (and rigid body dependent) parameters. Additionally the impact of the cranking model on the spin cutoff is an open field in which the cranking model would be very useful.

\footnotetext{
1 These are imposed, not obtained from systematics, the initial fragment spins are thus not affected by the change of model for the moment of inertia.
} 


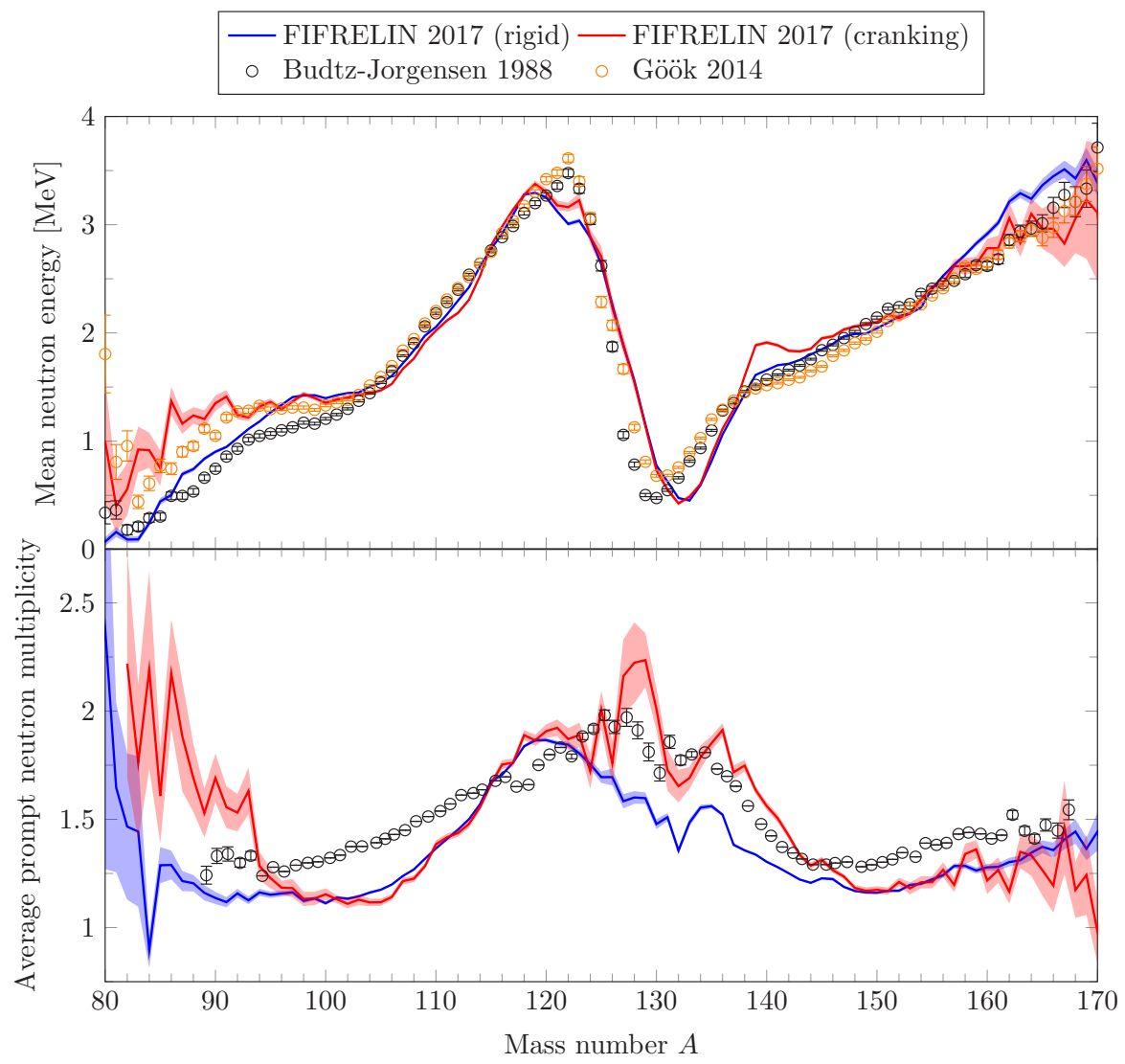

Fig. 2. Impact of the shell structure in rotational energy on the average neutron multiplicity (left) and the average neutron energy in center of mass (right).

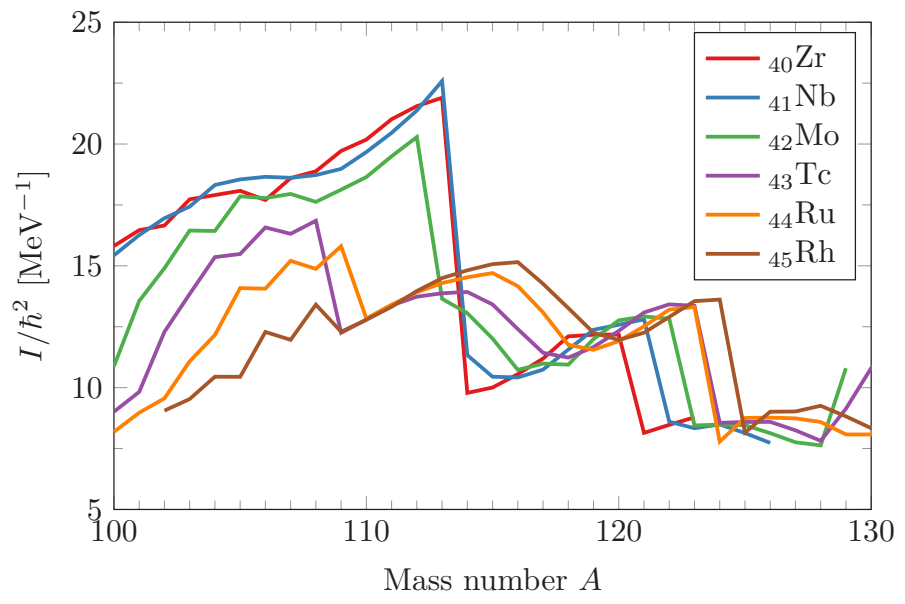

Fig. 3. Cranking moment of inertia for the light fragment region. 


\section{References}

1. Möller, P., Nix, J. R., Myers, W. D., and Swiatecki, W. J. (1995) Nuclear Ground-State Masses and Deformations. Atomic Data and Nuclear Data Tables, 59, 185.

2. Litaize, O. and Serot, O. (Nov, 2010) Investigation of phenomenological models for the Monte Carlo simulation of the prompt fission neutron and $\gamma$ emission. Phys. Rev. C, 82, 054616.

3. Capote, R., Herman, M., Obložinský, P., Young, P. G., Goriely, S., Belgya, T., Ignatyuk, A. V., Koning, A. J., Hilaire, S., Plujko, V. A., Avrigeanu, M., Bersillon, O., Chadwick, M. B., Fukahori, T., Ge, Z., Han, Y., Kailas, S., Kopecky, J., Maslov, V. M., Reffo, G., Sin, M., Soukhovitskii, Efrem, S., and Talou, P. (2009) RIPL - Reference Input Parameter Library for Calculation of Nuclear Reactions and Nuclear Data Evaluations. Nuclear Data Sheets, 110(12), 3107-3214 Special Issue on Nuclear Reaction Data.

4. Davies, K. T. R. and Nix, J. R. (Nov., 1976) Calculation of moments, potentials, and energies for an arbitrarily shaped diffuse-surface nuclear density distribution. Phys. Rev. C, 14, 1977-1994.

5. Bohr, A. and Mottelson, B. R. (1955) Moments of Inertia of Rotating Nuclei. Mat. Fys. Medd. Dan. Vid. Selsk., 30(1), 1-24.

6. Tamagno, P. Challenging fission cross section simulation with long standing macro-microscopic model of nucleus potential energy surface. PhD thesis Université de Bordeaux I (2015).

7. Inglis, D. R. (Sep, 1956) Nuclear Moments of Inertia due to Nucleon Motion in a Rotating Well. Phys. Rev., 103, 1786-1795.

8. Belyaev, S. (1959) Effect of Pairing Correlations on Nuclear Properties. Mat. Fys. Medd. Dan. Vid. Selsk., 31(11).

9. Bardeen, J., Cooper, L. N., and Schrieffer, J. R. (Apr., 1957) Microscopic Theory of Superconductivity. Phys. Rev., 106, 162-164.

10. Lipkin, H. J. (1960) Collective motion in many-particle systems: Part 1. The violation of conservation laws. Annals of Physics, 9(2), 272-291.

11. Möller, P. and Nix, J. (1992) Nuclear pairing models. Nuclear Physics A, 536(1), 20-60.

12. De Saint Jean, C., Habert, B., Litaize, O., Noguère, G., and Suteau, C. (2008) Status of CONRAD, a nuclear reaction analysis tool. EDP Sciences, 1, 251-254.

13. Bhat, M. R. (1992) Evaluated Nuclear Structure Data File (ENSDF). In Qaim, S., (ed.), Nuclear Data for Science and Technology, Research Reports in Physics pp. 817-821 Springer Berlin Heidelberg Data extracted using the NNDC On-Line Data Service from the ENSDF database, file revised as of (May 4, 2015).

14. Koning, A. J., Hilaire, S., and Duijvestijn, M. C. (2007) TALYS-1.0. In Bersillon, O., Gunsing, F., Bauge, E., Jacqmin, R., and Leray, S., (eds.), International Conference on Nuclear Data for Science and Technology - ND2007, Commissariat à l'Énergie Atomique Nice, France, April 22 27 Vol. 1, pp. 211-214.

15. Bečvaŕ, F. (1998) Simulation of $\gamma$ cascades in complex nuclei with emphasis on assessment of uncertainties of cascade-related quantities. Nuclear Instruments and Methods in Physics Research A, 417, 434-449.

16. Bethe, H. A. (Aug., 1936) An Attempt to Calculate the Number of Energy Levels of a Heavy Nucleus. Phys. Rev., 50, 332-341.

17. Gilbert, A. and Cameron, A. G. W. (1965) A composite nuclear-level density formula with shell corrections. Canadian Journal of Physics, 43(8), 1446-1496. 